\title{
Unfall- und Versicherungskunde
}

Blumenthal und Jaffè, Über Gewerbeekzem durch sog. Bakelitlack. Med. K1. 1929, Nr. 48, S. 1848.

Bakelitlack, eine Auflösung von Cumaronharz (Kondensations-produkt von Phenol und Formaldehyd) mit Terpentinöl verursacht bei Arbeitern und Arbeiterinnen eines Industriewerkes Ekzem der unbe-deckten Körperstellen u. a. im Gesicht und den Unterliedern mit diffuser Rötung und Schwellung. Der schädliche Stoff ist wahrscheinlich Phenol

(Phenolüberempfindlichkeit). Heilung nach Entfernung aus dem schä-digenden Arbeitsbetrieb.

\section{4}

Unfall- und Versicherungskunde.

Pollak, Lues und Trauma. Med. K1. 1929, Nr. 42, S. 1615.

Fall I. Bei einem 2ijährigen Rekruten führte ein nachweislich ganz leichter Stoß gegen den Kopf nach vier Tagen zu einem schweren als Hirnabszeß gedeuteten Krankheitsbild. Bei der Trepanation fand sich ein faustgroßes Hämatom in der Gegend der Zentralwindung mit beginnender Abszedierung. Exitus. - - Die ungewöhnliche Reaktion auf einen derartig kleinen Reiz führte zu der Feststellung, daß die Mutter des Pat. von ihrem an Paralyse gestorbenem Manne infiziert war. Im Blut und Liquor des Pat. Wa. positiv.

Fall II. 25Jährige Frau, die mit der rechten Kopfhälfte leicht an einen Balken streifte, nicht bewußtlos, nur etwas Brechreiz. Nach vier Tagen heftige Kopfschmerzen, Fieber, Doppelsehen, beiderseits Neuritis optica. Wa. in Blut und Liquor positiv. Der Ehemann, der an Tabes litt, hatte sie zwei Jahre nach einer luetischen Infektion geheiratet. Es wurde angenommen, daß die Lues bis zu dem Kopftrauma okkult verlief.

Fall III. 43Jährige Fabrikarbeiterin fünf Tage nach einer unge-wohnten Insolation des unbedeckten Kopfes Meningomyelitis. , Wa. im Liquor positiv, Pandy + + +, Goldsol; Lueszacke.

Nach Pollak ist die bisher von Wilson mit einer Woche angenommene Zeitspanne zwischen Unfall und Krankheitsausbruch zu kurz. Sie muß auf drei bis vier Wochen ausgedehnt werden. Das ist besonders bei Traumen geringfügiger Art notwendig, die zu ihrer Auswirkung längere Zeit brauchen.

E. Stoewer, Untersuchungsergebnisse über das Auftreten von professio-nellen Linsentrübungen bei $\Gamma$ euerarbeitern außerhalb der Glasindustrie.

Arbeitsschutz 1929, Nr. I-III, 5.

E. Stoewer, der durch seine Untersuchungen von 2000 Glasbläsern bekannte Verfasser, berichtet über 640, dem Feuer besonders ausgesetzte Industriearbeiter, bei denen trotz der Hilfsmittel der modernen Linsen-diagnostik nur in 0,8 \% eine gewerbliche Linsenschädigung festzustellen war. Wahrend gewerbliche Linsentrübungen bei Glasbläsern bis zu 100/0 vorkommen, sind sie bei Feuerarbeitern, die im Gegensatz zu Glas-arbeitern nur zeitweise der Strahlung ausgesetzt sind und vielfach einen Augenschutz tragen, relativ selten. Feuerstar wird meist nur bei älteren Leuten mit j ahrzehntelanger Berufsschädigung beobachtet. Trotzdem halt St. es für notwendig, daß der Gesetzgeber bei einer Neuregelung neben dem Glasbläserstar auch den in anderen 
Industriezweigen ent-stehenden Feuerstar in die entschädigungspflichtigen Berufskrankheiten einbezieht, da die Diagnose des gewerblich bedingten Feuerstars prak-tisch einwandfrei zu stellen ist. Die Dauer der Beschäftigung bis zum Auftreten von Linsentrübungen muß mit zehn Jahren als untere Grenze festgelegt werden. Charakteristisch sind die eigentümlichen Trübungen am hinteren Pol und Abspaltung der Zonula-Lamelle. Speichentrübungen sprechen nicht gegen Feuerstar. Die Abspaltung der Zonula-Lamelle ist ein so wichtiges Zeichen, daß es selbst bei voUentwickeltem Star, der sonst nicht von einem gewöhnlichen Altersstar abzugrenzen ware, ein-deutig für Berufsstar spricht.

Unfall- und Versicherungskunde.

125

Grad der Erwerbsunïähigkeit bei Verlust eines Auges nach G1/8wöhnung 25 v. H. - Keine Entschädigung für eine durch Vernachlässigung der Augenhöhle bedingte Eiterabsonderung. Der Hauer H. erlitt am 29. III. 1926 im Betriebe der Zeche H. einen komplizierten Bruch des rechten Gesichtsschädels, eine Zertrümmerung des rechten Auges und einen Oberkieferbruch. Das rechte Auge mußte entfernt werden. Für die Folgen des Unfalls wurde abwechselnd Vollrente und Heilanstaltspflege gewährt, vom 4. XL 1927 ab eine Dauer-rente in Höhe von 40 v. H. Diese wurde durch Bescheid vom 3. XI. 1928 mit Wirkung vom 1. I. 1929 auf 25 v. H. herabgesetzt, weil als alleinige Unfallfolge jetzt nur noch der Verlust des rechten Auges bestehe und An-passung an das einäugige Sehen eingetreten sei. Das Reichsversicherungs-amt hat diesen Bescheid in seiner Entscheidungvom 29. II. 1929 - la 2024/28/7 bestätigt - mit folgender Begründung:

,Nach ständiger Rechtsprechung des Reichsversicherungsamtes ist, wenn Gewöhnung an den durch den Verlust eines Auges bedingten Zustandeingetreten ist, in der Regel nur eine Minderung der Erwerbsfähigkeitvon 25 v. H. der Vollrente anzunehmen. Unter diesen Umständen mußdeshalb auch die von der Beklagten mit Bescheid vom 3. XL 1926 vorgénommene Herabsetzung der Rente von 40 auf 25 v. H. der Vollrentefür die Zeit nach dem 1.1.1929 als ausreichende Entschädigung angesehenwerden; denn diese Herabsetzung ist fast drei Jahre nach dem Unfallerfolgt, so daß ohne Bedenken angenommen werden kann, daß der Ver-letzte sich in dieser Zeit an das einäugige Sehen so vollkommen gewöhnthat, daß eine höhere Beschränkung der Erwerbsfähigkeit als die normalebei ihm nicht mehr vorliegt. Der Umstand, daß die Augenhöhle nochEiter absondert, vermag eine höhere Rente nicht zu rechtfertigen; denndiese Eiterung ist nicht auf den Unfall, sondern auf Vernachlässigungder Augenhöhle durch den Kläger zurückzuführen. Wie das Reichsver-sicherungsamt ständig entschieden hat (E 2772 I AN, 1915 S. 322) sinddie Verletzten verpflichtet, zur möglichsten Beseitigung der Unfallfolgenbeizutragen. Tun sie dies nicht, so können sie für die dadurch bedingtenUnfallfolgen Entschädigung nicht verlangen." Heßberg (Essen).

Buchbesprechung.

Das Quarzlicht und seine Anwendung in der Medizin. Von F. Thedering.

7. Auflage. Oldenburg i. O. Gerhard Stalling.

I. Im ersten

allgemeinen Teil seines Buches gibt der Verfasser zu-nächst einen Überblick über die Entwicklung der Strahlenkunde und über die Art und Wirkungsweise der Quarzlampen.

Berücksichtigung finden auch die neueren Errungenschaften in der Ultravialettmessung sowie un-sere Kenntnis über chemische und biologische Lichtwirkungen. Je ein besonderer Abschnitt behandelt die Pigmentbildung sowie die sonstigen Veränderungen 
welche das Licht an der Haut und durch deren Vermitt-lung im Körper hervorruft; weiterhin wird die allgemeine Technik der Lichtbehandlung besprochen sowie die Nutzanwendung des Quarzlichtes 